\title{
Advancing Research in the Era of Healthcare Reform The 19th Annual HMO Research Network Conference, April 16-18, 2013, San Francisco, California
}

\author{
Laurel A. Copeland, PhD and John E. Zeber, PhD
}

\author{
Corresponding Author: \\ Laurel A. Copeland, PhD \\ Center for Applied Health Research \\ Scott \& White Healthcare, jointly with \\ Central Texas Veterans Health Care System \\ 2102 Birdcreek Dr \\ Temple, TX 76502 \\ Tel: (254) 2I5-9880 \\ Fax: (254) 77I-082। \\ Email: Icopeland@sw.org \\ Keywords: \\ Biomedical Research \\ Congresses as Topic \\ Health Maintenance Organizations \\ Humans \\ Quality of Health Care \\ Received: May 17, 2013 \\ Accepted: May 23, 2013 \\ doi: $10.3121 / \mathrm{cmr} .2013 .1175$
}

$\mathrm{H}$ ealthcare system transformation has pursued higher quality, more affordable healthcare from the popularization of health maintenance organizations (HMOs) in the 1970s, through the innovations in structure and management of the 1990s, to the current-day challenge of extending healthcare coverage to previously uninsured groups as partially conceptualized in the Patient Protection and Affordable Care Act (PPACA) toward Accountable Care Organizations (ACOs). Census 2010 reported that 17\% of Americans had no insurance whatsoever, $18 \%$ were publicly insured, ${ }^{1}$ and the remaining $65 \%$ were privately insured. Overall, approximately 1 in 4 Americans is enrolled in an HMO, including Medicare HMO options.

Research on persons covered by managed care insurance and integrated delivery systems is needed to round out our understanding of how people use healthcare and how their health status relates to that usage. These are the middle class Americans, the working well, who are not well represented in federal programs. Groups commonly examined by health services researchers include Medicaid beneficiaries (impoverished, mostly children), Medicare beneficiaries (older or disabled persons), and Veteran's Administration patients (disabled, impoverished, tied to military recruitment trends, mostly male). Other major sources of health data are primarily survey-based (such as the National Ambulatory Medical Care Survey on outpatient care) or topically focused (Surveillance Epidemiology and End Results on cancer, Health Cost and Utilization Project on inpatient experiences) and therefore lacking in comprehensiveness and in detail regarding specific procedures, medications, and diagnoses received. These factors - population and data qualityspurred the development of the HMO Research Network (HMORN) and its Virtual Data Warehouse (VDW), a clinical records-based data resource on insured persons. Now in its $19^{\text {th }}$ year, the HMORN held its annual meeting in San Francisco, April 16-18, 2013, hosted by Kaiser Permanente of Northern California with Dr. Alan Go as Chair.

We are now in the era of healthcare reform. Cost containment continues to pressure development of efficient care delivery and payment structures, with quality of care and patient experience as equal goals in the "Triple Aim". ${ }^{2}$ In the past two years, changes enacted via the American Recovery and Reinvestment Act-funded Health Information Technology for Economic and Clinical Health electronic medical records adoption mandate (beginning in 2011), expansion initiatives for Medicaid, rural and other underserved populations (as of March 2012), and economic market forces, in general, have continued to shape both clientele-served and cost reimbursement schemas. This evolution has led healthcare systems to ask, why do people come through our doors? Who chooses not to return? What do we have to do as a system to provide high quality care at a sustainable price, engendering excellent patient satisfaction? These challenges informed the theme of the 2013 
HMO Research Network Conference, "Advancing Research in the Era of Healthcare Reform."

\section{The State of the Network}

John Steiner, Senior Director of Kaiser Permanente Colorado Institute for Health Research and Chair of the HMORN Governing Board, delivered his report on the state of the HMO Research Network to kick off the meeting. The Network now comprises 18 sites with 1450 research investigators and staff, having elevated three associate member sites to full member status and lost one member when its mission evolved to a statewide role. The VDW now holds multiple years of data; the 18 sites represent 16 million covered lives. Research productivity metrics in the past year were impressive with over 2000 papers published by HMORN-based scientists; the Cancer Research Network and Vaccine Safety Datalink were renewed; the National Institutes of Health (NIH) Health Care System Research Collaboratory for large-scale clinical trials was initiated; and seven pragmatic trials received planning grant funds (of which 3 were led by HMORN investigators and 2 others with HMORN participation).

American Recovery and Reinvestment Act funding will end within the calendar year, and other funding sources, notably $\mathrm{NIH}$, have already shrunk. HMORN investigators will deal with scarce resources and new funding mechanisms such as indefinite quantity/indefinite duration awards to meet funder needs more efficiently. Where are we going, and how will we get there? By putting forward our prime assets: researchers embedded in healthcare delivery systems, rich clinical data, a network of ready and able colleagues, and the ability to learn from our data collaboratively across HMORN sites and with non-HMORN partners, we will add value to health care reform.

As part of the roadmap, the HMORN will devote resources to expanding our communication strategy, nurturing new internal and external partners, promoting cluster randomized trials and pragmatic trials, and fostering the HMORN culture of teamwork with respect for autonomy and transparency. Maintaining research embedded within managed care systems will continue to distinguish HMORN investigators from other scientists, and HMORN will be uniquely positioned to promote the development of true learning healthcare systems. Here is the take-home message: the HMORN is eager to work with researchers, practitioners, and healthcare leadership. Internally, HMORN will expand its data capabilities and knowledge management to optimize transmission of new knowledge while avoiding redundancy, both in research and in translation into practice. Efficiency at all levels will be our mantra.

\section{Early Career Investigators}

In the awards session, four Early Career Investigators were recognized, having been selected via a competitive evaluation overseen by Dr. Alyse Adams based on abstracts and letters of support. Each awardee presented his research. Dr. Darren Toh, Harvard Pilgrim Health Care, spoke of his work in his presentation titled "Confounding Adjustment in Distributed Data Networks without Sharing of Individual-Level Data," focusing on the methods. He demonstrated that we can conduct adjusted regression modeling without person-level HMORN data, an important capacity that facilitates multisite collaborations.

Next, Dr. David D. McManus, Fallon Community Health Plan/ Reliant Medical Group, presented "Atrial Fibrillation and Outcomes in Heart Failure with Preserved Versus Reduced Left Ventricular Ejection Fraction." McManus worked with the Cardiovascular Research Network (CVRN) team. The CVRN has constructed a deeply detailed surveillance database incorporating clinical and administrative data with chart review. CVRN searched imaging reports and echocardiogram results to populate indicators on 23,644 patients. Among other things, this study illustrated that application of rigorous methods using chart review and natural language processing is feasible and fruitful within HMORN studies.

The third recipient was Dr. Christine Y. Lu, Harvard Pilgrim Health Care, who presented her study, "Changes in Antidepressant Utilization and Suicide Attempts Following the 2004 FDA Black Box Warning and Media Coverage.” Working with data from the 11 sites in the Mental Health Research Network, Dr. Lu applied an interrupted time-series design and segmented regression analysis. The study demonstrated the unparalleled utility of HMORN for post-marketing surveillance on rare outcomes.

Last among the Early Career Investigators was Dr. Powell Jose from the Palo Alto Medical Foundation for Health, Research, and Education. Dr. Jose described his work, "CulturallyCompetent Heart Health Coaching Improves Lipids in South Asians." One of the many advantages of HMORN is the ability to access diverse patient samples including racial/ethnic subgroups.

\section{The Plenary Session}

Mark McClellan, MD, PhD, MPA, Director of the Engelberg Center for Health Care Reform and Leonard D. Schaeffer Chair in Health Policy Studies at The Brookings Institution, was the keynote speaker. His summary of the current health policy environment touched rapidly on federal spending, sequestration, healthcare reform, learning healthcare systems, and the value Americans place on survival. Federal expenditure on healthcare over the past 45 years has shown tremendous growth while spending on infrastructure, military, support services, and Social Security has remained stable. McClellan explained that because Americans value preserving life through healthcare so highly, it is necessary to find a sustainable solution to uncontrolled healthcare costs that achieves better care at the same or lower cost. Rationing healthcare will fail.

The HMORN can fit into this paradigm by increasing the rate and quality of information return to our host healthcare systems to create true learning healthcare systems, to foster 
truly patient-centered care, to reduce gaps in prevention, to improve knowledge management and decision support, and to increase healthcare efficiency through creative quality-linked care and payment schemas. HMORN can also help educate the public on the changes and choices the Affordable Care Act is bringing, a monumental outreach task. In addition, reduced payments to inpatient care facilities will force development of new ways to care for the critically ill and those unable to manage at home. HMORN must participate in finding solutions to the problems the proposed cuts will create. Finally, HMORN can advance personalized medicine through genomics research and the study of crafting targeted drug treatments. Personalized medicine relies on the innumerable variations present in our genes and arising over our lifetimes as mutations accumulate. Therefore, very large networks facilitate progress in a realm where even "common" diseaseassociated variations are rare.

HMORN can contribute to policy reform by showing, incontrovertibly and with large numbers of patients and systems, which policies are counter-productive. McClellan offered the example of perverse incentives penalizing many lower-cost/higher-quality care options; such findings need to be persuasively and widely disseminated, and policy adjusted accordingly. Accountability and reforms must be applied to care delivery, coverage policies, and also to drugs and devices. We will need to be able to persuade patients when their costly treatment preferences are not worth it. Results-based payments may apply to high-cost biologics and diagnostics. We need to develop and demonstrate a more routine way to set up registries to track devices and their outcomes; CVRN has begun this type of work.

According to McClellan, the HMORN can provide muchneeded and better data on innovations to improve adherence to treatments such as wireless pill bottles, pedometers, and scales. Incentives for healthier behavior must be created and tested-McClellan spun off a list of possibilities: daily lotteries for good adherence, having "deposit accounts" with funds at risk if the patient is non-adherent, adding a social component to share experiences or to engage the patient's support network in automated alerts, even team competitions in adherence. Other areas ripe for HMORN work might include predicting high cost members of health plans, demonstrating the value of limiting switching among plans, using distributed analysis to support care delivery decisions or enhance quality and safety reporting. The Mini-Sentinel initiative provides a model of this, offering rapid responses to Food and Drug Administration queries about drug and device safety. HMORN could also help the host organizations make coverage decisions based on comparative effectiveness analyses; this is already being done in Europe. Other potential uses of the HMORN VDW include understanding regional healthcare system performance, clinical registries, customized cloud-based networks instead of separate VDW's for research, and integrating the network data with care delivery and quality reporting.
Overall, the HMORN $19^{\text {th }}$ annual meeting welcomed 424 attendees including 16 non-HMORN members. In addition to the highlighted talks, a total of 52 oral presentations were given across a range of important clinical topics. These included thematic sessions on Health Informatics, HMORN Administrative and Technical Issues, Mental Health and Chemical Dependency, the VDW, and Pharmacoepidemiology. During three widely attended sessions, 171 posters were on display, focusing on cancer, chronic disease, health services and policy, genetics, comparative effectiveness research, and other diverse clinical topic areas. There were also 52 ancillary meetings held throughout the conference, from the HMORN Governing Board to the major sub-networks (eg, the Mental Health, Cancer, and Cardiovascular Research Networks), with additional meetings bringing together collaborators to discuss special projects on targeted research efforts such as rural health issues, aging, and obesity.

\section{Unanswered Questions}

The $19^{\text {th }}$ Annual HMO Research Network Conference has concluded, and as we head back to our host organizations and tightening budgets, we have little time to address very pressing questions. How can we optimize collaboration and standardization? Can we collaborate with other consortia such as the Blues and with the Veterans Health Administration to produce research on a large proportion, even a majority, of Americans? How shall we attract external principal investigators to write HMORN investigators and staff into their proposals so that we can optimize this remarkable resource? How can we bring ourselves and our host organizations to view a cloudbased data solution as desirable, even necessary? How will we continue to improve our integration with clinical care? Some ideas floated at this meeting included providing daily reports to clinics about the number of infectious disease cases in the system; registries for diabetes, weight management, mental health, pediatric health, obstetric health; clinic-specific and provider-specific quality metrics (ie, performance measures such as number of diabetic patients who had eye/foot exams past year) to keep clinicians updated on patient profiles and treatment trends - possibly in the context of cost variation as well. The true goal of a cloud-based VDW would entail merging real identifiers to capture each person's comprehensive healthcare history without divulging contributing sites to one another. Only a fully identified merge platform would address complaints that out-of-system use and comorbidity is not captured with currently available methods. Expanding and innovating our data capabilities and communication strategies, and collaborating with the widest possible range of entities, will keep the HMORN on the leading edge of research in the era of healthcare reform.

\section{References}

1. O'Hara B, Caswell K. Health status, health insurance, and medical services utilization: 2010. Curr Pop Rep 2012: 70-133. Available at: http://www.census.gov/prod/2012pubs/ p70-133.pdf. Accessed April 24, 2013.

2. Berwick DM, Nolan TW, Whittington J. The triple aim: care, health, and cost. Health Aff (Millwood) 2008;27:759-769. 